\title{
PROFILO DI PAZIENTI VISITATI IN UN AMBULATORIO DI GENETICA MEDICA IN UN CENTRO UNIVERSITÁRIO DE BELÉM, PARÁ, AMAZZONIA
}

\section{ARTICOLO ORIGINALE}

LIMA, Gabriela Elenor dos Santos ${ }^{1}$, MARTINS, Carlos Henrique Lopes$^{2}$, DENDASCK, Carla Viana ${ }^{3}$, OLIVEIRA, Ciane Martins de ${ }^{4}$, OLIVEIRA, Euzébio de ${ }^{5}$

LIMA, Gabriela Elenor dos Santos. Et. Profilo di pazienti visitati in un ambulatorio di genetica medica in un Centro Universitário De Belém, Pará, Amazzonia. Revista Científica Multidisciplinar Núcleo do Conhecimento. Anno 06, Ed. 03, Vol. 11, pp. 48-62. marzo 2021. ISSN: 2448-0959, Link di accesso: https://www.nucleodoconhecimento.com.br/salute/ambulatorio-di-genetica, $\quad$ DOI: 10.32749/nucleodoconhecimento.com.br/salute/ambulatorio-di-genetica

\section{RIEPILOGO}

La Genetica Medica (GM) è diventata una specialità medica riconosciuta, con concetti e approcci importanti nella diagnosi e nel trattamento di molte malattie comuni e rare. Le malattie genetiche seguono i modelli ereditari e possono essere autosomi recessive, autosomiche dominanti, legate al cromosoma $\mathrm{X} \mathrm{o}$ al cromosoma Y, o multifattoriali. Lo scopo di questo studio era determinare il profilo dei pazienti trattati in una clinica ambulatoriale GM in un centro universitario di Belém, nello stato di Pará. I dati sono stati raccolti dalle cartelle cliniche dei pazienti, visti tra il 2014 e il

\footnotetext{
${ }^{1}$ Accademico medico presso il Centro Universitario dello Stato di Pará - CESUPA.

2 Studente di medicina presso il Centro Universitario dello Stato di Pará - CESUPA.

${ }^{3}$ Teologo, Dottorato di Ricerca in Psicoanalisi Clinica. Lavora da 15 anni con metodologia scientifica (metodo di ricerca) nella guida alla produzione scientifica di studenti di master e dottorato. Specialista in Ricerche di Mercato e Ricerca focalizzato sulla salute.

${ }^{4}$ Dottorato di ricerca in Genetica Medica. Professore e Ricercatore presso il Centro Universitario dello Stato di Pará (CESUPA).

${ }^{5}$ Dottorato di ricerca in Medicina/Malattie Tropicali. Professore e ricercatore presso I'Università federale di Pará - UFPA.
}

RC: 79876

Disponível em: https://www.nucleodoconhecimento.com.br/salute/ambulatorio-di- 
2019, utilizzando il questionario dei ricercatori, con dati analizzati e tabulati attraverso il programma Microsoft Excel. Sono state analizzate in totale 101 cartelle cliniche, con predominanza di femmine (51 pazienti). Inoltre, la maggior parte delle cure è stata per i bambini $(41,5 \%)$. Per quanto riguarda l'etnia, sono state osservate solo le variabili "bianco" e "marrone", con una maggiore prevalenza di pazienti marroni (78 del totale). Inoltre, Belém era la città più diffusa nella naturalezza dei pazienti (61 record). Le specialità con il maggior numero di rinvii alla clinica ambulatoriale GM erano endocrinologia e neurologia, con il ritardo dello sviluppo neuropsicomotorio come diagnosi più frequente. In 42 cartelle cliniche, l'età alla diagnosi non era presente. Dei 101 pazienti, solo 16 avevano consulenza genetica e nei restanti 85 non c'era traccia di queste informazioni. Infine, l'età materna alla nascita non è stata riscontrata nella maggior parte delle cartelle cliniche (assente nel $61,38 \%)$. Pertanto, è importante sviluppare un profilo del paziente visto in una clinica ambulatoriale GM, poiché diventa possibile identificare eventuali guasti nel servizio fornito, oltre ad adattare la relazione medico-paziente.

Parole chiave: Genetica medica, clinica ambulatoriale, malattie genetiche.

\section{INTRODUZIONE}

La genetica emerse in medicina all'inizio del XX secolo, quando si percepiva che le leggi mendeliana dell'ereditarietà potevano spiegare la recidiva di alcuni disturbi familiari. Nei successivi 100 anni, medical genetics (GM) ha cessato di essere una piccola sottospecialità focalizzata solo su pochi rari disturbi ereditari, per diventare una specialità medica riconosciuta, con concetti e approcci importanti nella diagnosi e nel trattamento di molte malattie, sia comuni che rare (NUSSBAUM et al., 2016).

GM è stata riconosciuta come specialità medica dal Federal Council of Medicine nel 1983 e nel 1986, per espandere la conoscenza della specialità, è stata creata la Società Brasiliana di Genetica Medica. Più recentemente, GM ha agito in modo

RC: 79876

Disponível em: https://www.nucleodoconhecimento.com.br/salute/ambulatorio-di- 
pertinente nella consulenza genetica (SOCIEDADE BRASILEIRA DE GENÉTICA MÉDICA, 2020).

Secondo l'Organizzazione Mondiale della Sanità (OMS), la consulenza genetica riguarda un chiarimento genetico, che mira a prevenire genotipi responsabili di malattie o difetti alla nascita, attraverso l'identificazione prospettica o retrospettiva di unioni in grado di produrre questi cambiamenti. Inoltre, la consulenza copre anche la segnalazione di problemi legati all'insorgenza o al rischio di una malattia genetica che si verifica in famiglia. Pertanto, è necessario partecipare a un team multidisciplinare di professionisti in grado di consigliare il paziente e la sua famiglia in merito alla diagnosi, al decorso della malattia, agli approcci disponibili, al rischio di recidiva e alle alternative a tale rischio. In questo contesto, si evidenzia che il professionista che offre consulenza non può suggerire, indicare o richiedere comportamenti ai pazienti da lui trattati (BERTOLLO et al., 2013).

Una malattia di origine genetica ha origine da cambiamenti nelle sequenze di Acido Deossiribonucleico (DNA), il materiale genetico di ogni individuo o mutazioni a livello di cromosomi sessuali o autosomali (GRIFFITHS; WESSLER; DOEBLEY, 2016).

Le malattie genetiche seguono modelli di ereditarietà, possono essere: (1) recessive autosomiche, che si manifestano solo in individui omozigoti per un gene autosomico, cioè gli individui affetti hanno due copie del gene mutante, come la fibrosi cistica, che risulta da una mutazione nel gene CFTR sul cromosoma 7, e la fenilchetonuria classica, correlata alle mutazioni nel gene situato sul cromosoma 12; (2) dominante autosomica, in cui i geni mutanti si trovano anche su cromosomi autosomi, tuttavia, una singola copia genica è in grado di causare la malattia, come la malattia di Huntington; (3) Legati all'X, noti anche come ereditarietà legate al sesso, in questo caso $\mathrm{i}$ cromosomi si trovano in una regione non omologha del cromosoma $\mathrm{X}$ e le mutazioni sono dominanti nei maschi, gli esempi principali sono il daltonismo, l'emofilia e la distrofia muscolare di Duchenne; (4) legato all'ereditarietà o all'ereditarietà di $Y$, o holândrica o ereditarietà limitata al sesso, in cui l'insorgenza

RC: 79876

Disponível em: https://www.nucleodoconhecimento.com.br/salute/ambulatorio-di- 
della malattia è correlata al cromosoma $Y$ nel suo cromosoma $x$ non omologo; e (5) poligenici o multifattoriali, che derivano da mutazioni in diversi geni o derivano dall'interazione di diversi fattori ambientali, da qui il momento clou per l'Amazzonia del Brasile, a causa della sua grande peculiarità e diversità di ambienti ecologici e non ecologici che sono direttamente collegati alla vita delle persone e possono contribuire in modo significativo ai cambiamenti fenotipici in più geni , ad esempio, malattie coronariche, cancro e schizofrenia (LOBO, 2008; BERTOLLO et al., 2013; NUSSBAUM et al., 2016).

Le malattie genetiche possono anche derivare da alterazioni cromosomiche, che sono classificate come: (1) mutazioni numeriche, in cui vi è un cambiamento nel numero di cromosomi nella cellula; e (2) mutazioni strutturali, in cui c'è un cambiamento nella forma o nelle dimensioni dei cromosomi. Ci sono anche casi in cui può verificarsi una miscela di linee cellulari, con la presenza di un lignaggio normale e un altro alterato, un evento chiamato mosaicismo (BORGES-OSÓRIO; ROBINSON, 2013).

Le variazioni cromosomiche del carattere numerico sono classificate in due categorie principali: euploidie e aneuploidie. Le euploidie coinvolgono interi genomi, che colpiscono tutti i cromosomi di un individuo, essendo una condizione incompatibile con la vita. Gli aneuploidi comportano cambiamenti nel numero cromosomico, con aumento o perdita di uno o più cromosomi, senza coinvolgere l'insieme aploide (JORDE; CAREY; BAMSHAD, 2016).

Negli uomini, questi cambiamenti cromosomici, specialmente nei cromosomi più grandi, sono quasi sempre letali, in modo diverso da ciò che accade sui cromosomi più piccoli, come il cromosoma 21, che può rimanere nel lignaggio cellulare, sebbene si traduce in nascite con malformazioni fisiche e mentali. Tra le conseguenze generate dalle alterazioni cromosomiche, ci sono le sindromi di Down, Edwards, Patau e Klinefelter (ZANELLA; TERENCIO, 2016).

RC: 79876

Disponível em: https://www.nucleodoconhecimento.com.br/salute/ambulatorio-di- 
Da quanto sopra, vale la pena ricordare che, poiché le malattie genetiche sono una condizione non curata, il follow-up è una parte essenziale del trattamento e dura per tutta la vita del paziente. Pertanto, il presente studio mira a determinare il profilo dei pazienti visti in una clinica ambulatoriale GM, in un Centro Universitario di Belém, Pará, Amazzonia.

\section{MATERIALE E METODO}

Si tratta di uno studio descrittivo e retrospettivo, in cui le cartelle cliniche dei pazienti trattati da gennaio 2014 a marzo 2020 sono state analizzate presso la clinica ambulatoriale GM del Center for Medical Specialties di un centro universitario situato nella città di Belém, Pará. Tutti i pazienti (mantenuto il loro anonimato) hanno firmato un modulo di autorizzazione in modo che le rispettive cartelle cliniche potessero essere analizzate, per la raccolta dei dati, per lo sviluppo di questa ricerca. II progetto è stato presentato e approvato dal Comitato Etico per la Ricerca con gli Esseri Umani, secondo il parere di approvazione del numero 2.917.287. Oltre al protocollo di questo studio ha seguito rigorosamente le norme della Risoluzione n. 466/12 del Consiglio Sanitario Nazionale del Brasile (BRASIL, 2012), che stabilisce standard per la ricerca che coinvolge esseri umani.

Sono state incluse le cartelle cliniche dei pazienti trattati solo durante il periodo indicato per questa ricerca e sono state escluse le cartelle cliniche dei pazienti osservati in cliniche ambulatoriali di altre specialità mediche. Il profilo di ogni paziente è stato analizzato per le seguenti variabili: sesso, età, etnia, origine, origine del rinvio medico, diagnosi, età alla diagnosi, età della madre alla nascita, complicanze in gravidanza, consulenza genetica e follow-up da parte del team multidisciplinare.

I pazienti sono stati classificati in base all'età, secondo i criteri dell'OMS, nei bambini (fino a 9 anni), negli adolescenti (dai 10 ai 19 anni), negli adulti (dai 20 ai 59 anni) e negli anziani (dai 60 anni).

$\mathrm{RC}: 79876$

Disponível em: https://www.nucleodoconhecimento.com.br/salute/ambulatorio-di- 
La raccolta dei dati, basata sull'analisi delle cartelle cliniche, è stata eseguita utilizzando il questionario dei ricercatori, contenente domande oggettive e soggettive. I dati sono stati quindi tabulati e analizzati con l'aiuto del programma Microsoft Excel 2016.

\section{RISULTATI}

Nel corso del periodo sono state analizzate 101 cartelle cliniche. Di questi, 49 erano pazienti maschi e 51 femmine (figura 1). Inoltre, si è riscontrato che la maggior parte delle visite è stata per bambini, con il $41,5 \%$ del totale, seguita dal $31,68 \%$ per gli adolescenti e dal 20,79\% per gli adulti. Gli anziani rappresentavano la percentuale più bassa, con il 5,94\% delle visite (figura 2). Vale la pena ricordare che, del numero totale di bambini, la frequenza più alta delle registrazioni è stata di pazienti di 4 anni, riscontrata nell'analisi di 10 cartelle cliniche. Nelle altre fasce d'età, la distribuzione tra le età era simile.

RC: 79876

Disponível em: https://www.nucleodoconhecimento.com.br/salute/ambulatorio-di- 
Figura 1: Distribuzione dei pazienti osservati presso la clinica ambulatoriale di genetica medica per genere.

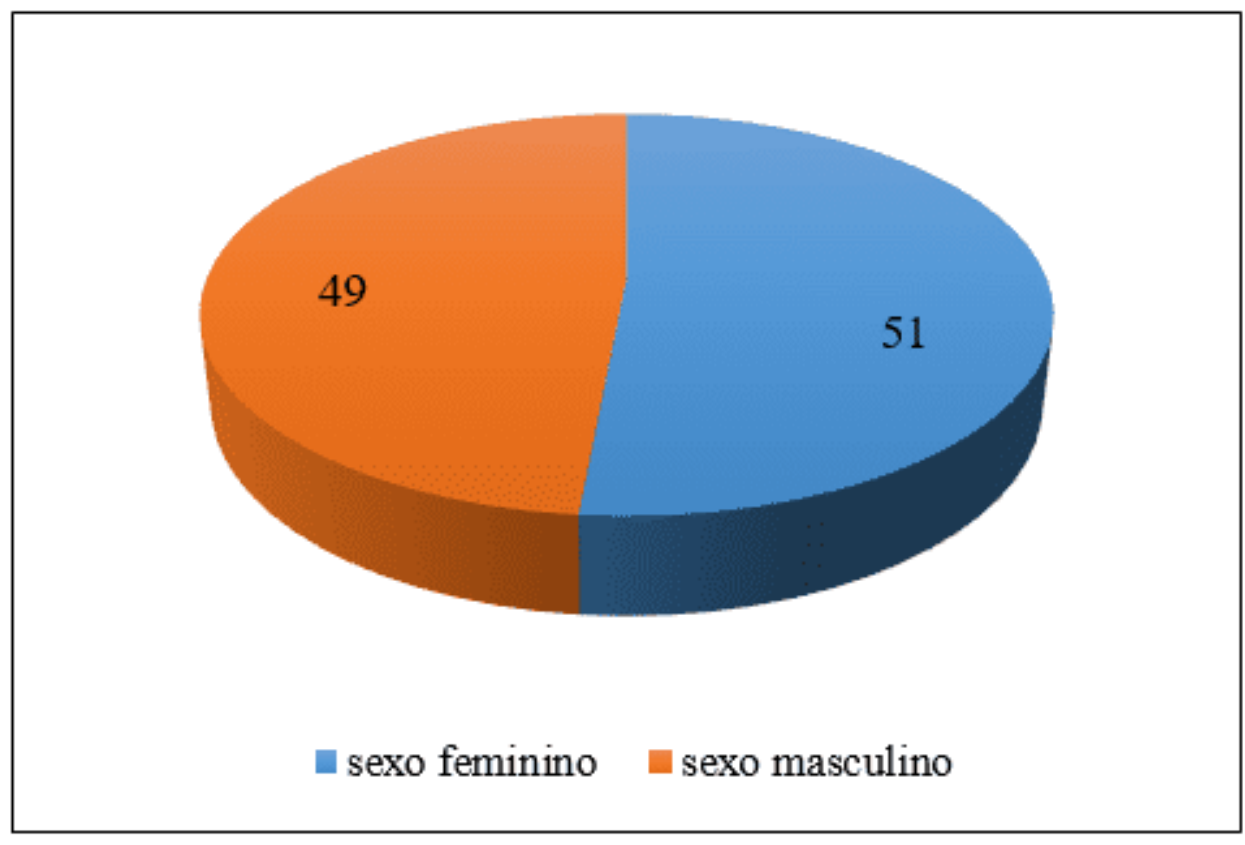

Fonte: Dati di ricercar.

RC: 79876

Disponível em: https://www.nucleodoconhecimento.com.br/salute/ambulatorio-digenetica 
Figura 2: Distribuzione dei pazienti osservati presso la clinica ambulatoriale di genetica medica, per fascia d'età.

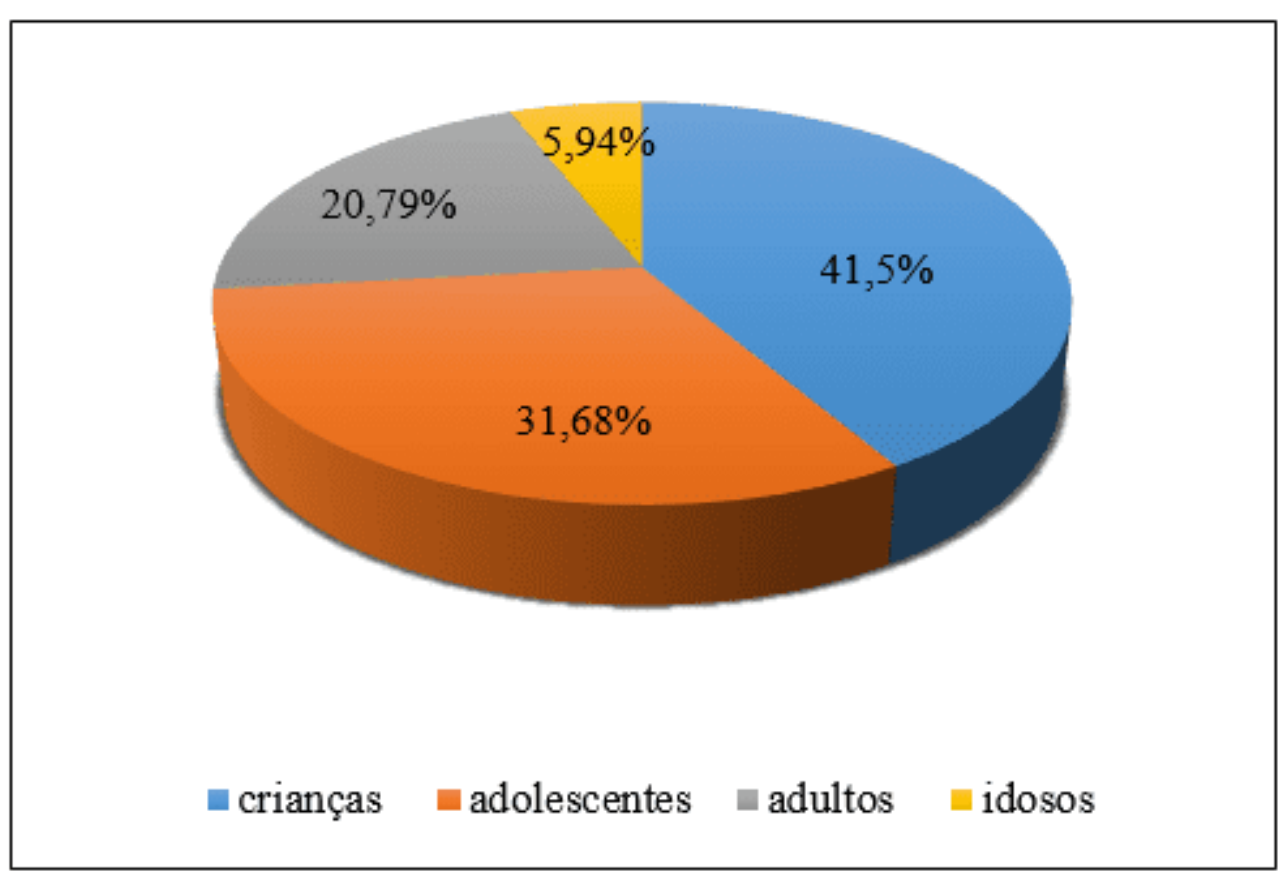

Fonte: Dati di ricerca.

Per quanto riguarda l'etnia, durante l'analisi delle cartelle cliniche, sono state trovate le variabili "bianco" e "marrone", senza precedenti di pazienti neri, oltre ad avere cartelle cliniche senza compilare la domanda. Pertanto, c'erano 10 pazienti bianchi, 78 pazienti marroni e 13 in cui le informazioni non erano incluse.

Belém era la città più diffusa nella naturalezza dei pazienti. La capitale del Pará concentrò 61 record, seguiti da Abaetetuba con 7 pazienti. Si sottolinea che la clinica ambulatoriale frequentata nel periodo analizzato, per lo più città che compongono la regione metropolitana di Belém. Solo 1 caso è stato un'eccezione: quello di un paziente citato dal servizio di Genetica di un'università di Campinas, nello stato di San Paolo. Altre città trovate nello studio sono state Altamira (1), Ananindeua (4), Barcarena (3), Benevides (1), Bragança (3), Cametá (4), Capanema (1), Castanhal

RC: 79876

Disponível em: https://www.nucleodoconhecimento.com.br/salute/ambulatorio-di- 
(2), Cotijuba (1), Marabá (1), Marituba (1), Mosqueiro (2), Ourém (1), Paragominas (3), Portel (1), Santo Antônio do Tauá (1), Tucuruí (1) e Vigia (1) (Figura 3).

Figura 3: Distribuzione dei pazienti osservati presso la clinica ambulatoriale di genetica medica, per origine.

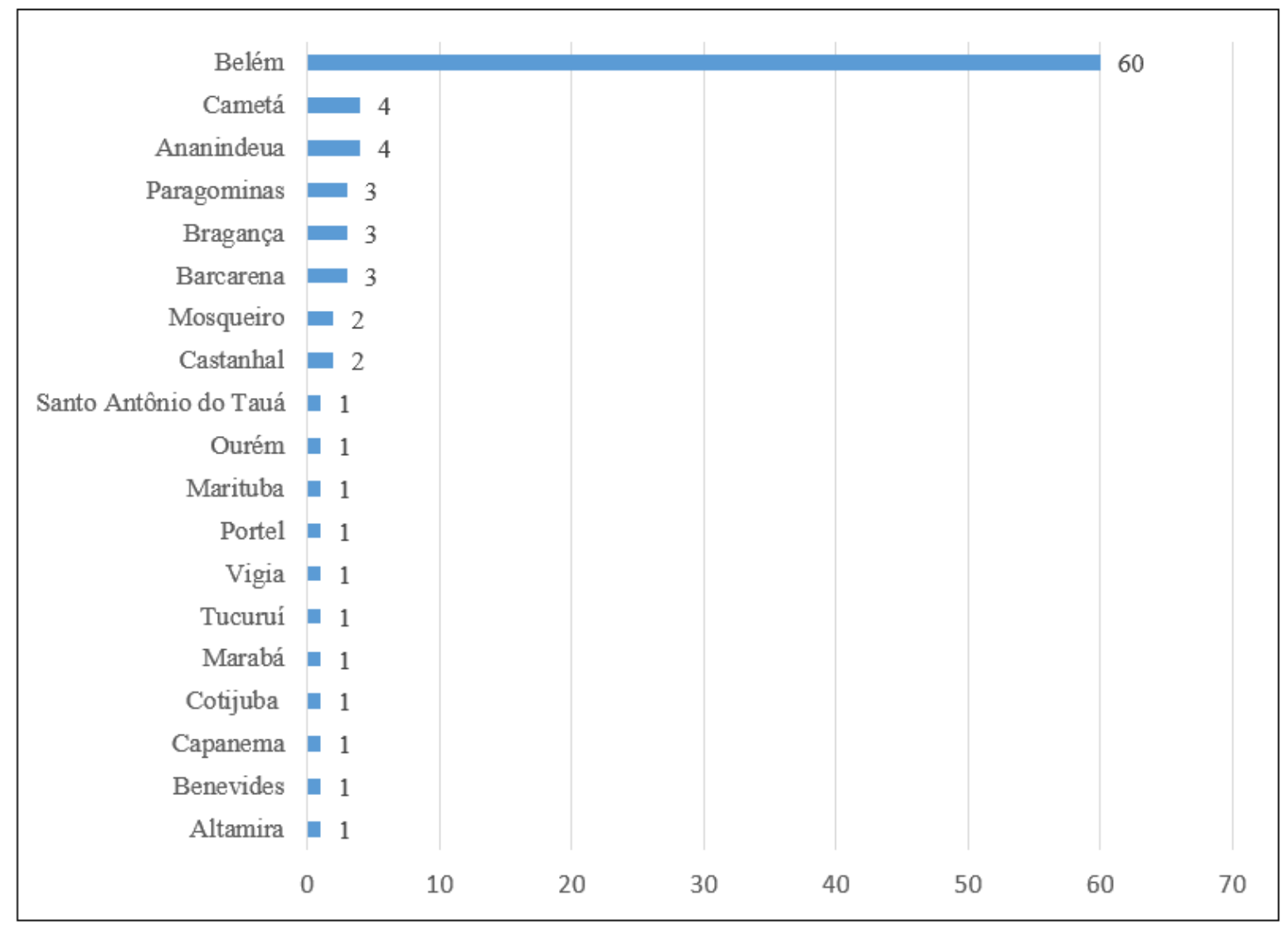

Fonte: Dati di ricerca.

È stato anche scoperto che endocrinologia e neurologia sono le specialità con il maggior numero di rinvii medici alla clinica ambulatoriale GM. La neurologia ha riguardato 26 pazienti, con una predominanza di bambini, 25 dei quali pazienti pediatrici $(10,89 \%)$ e solo 1 adulto. L'endocrinologia ha inviato 22 pazienti. Tuttavia, vi è anche un gran numero di cartelle cliniche in cui queste informazioni non sono state trovate, per un totale del $26,73 \%$. Oltre a questi, altre specialità mediche che si

RC: 79876

Disponível em: https://www.nucleodoconhecimento.com.br/salute/ambulatorio-digenetica 
riferivano al GM erano ginecologia (2), mastologia (2), neonatologia (2), medicina clinica (1), dermatologia (1), gastropediatria (1), genetica della città di Campinas (1), ortopedia (1), pulmonologia (1), reumatologia (1), salute degli anziani (1) e unità sanitaria di base (1) (figura 4).

Figura 4: Distribuzione dei pazienti osservati presso la clinica ambulatoriale di genetica medica, con rinvio.

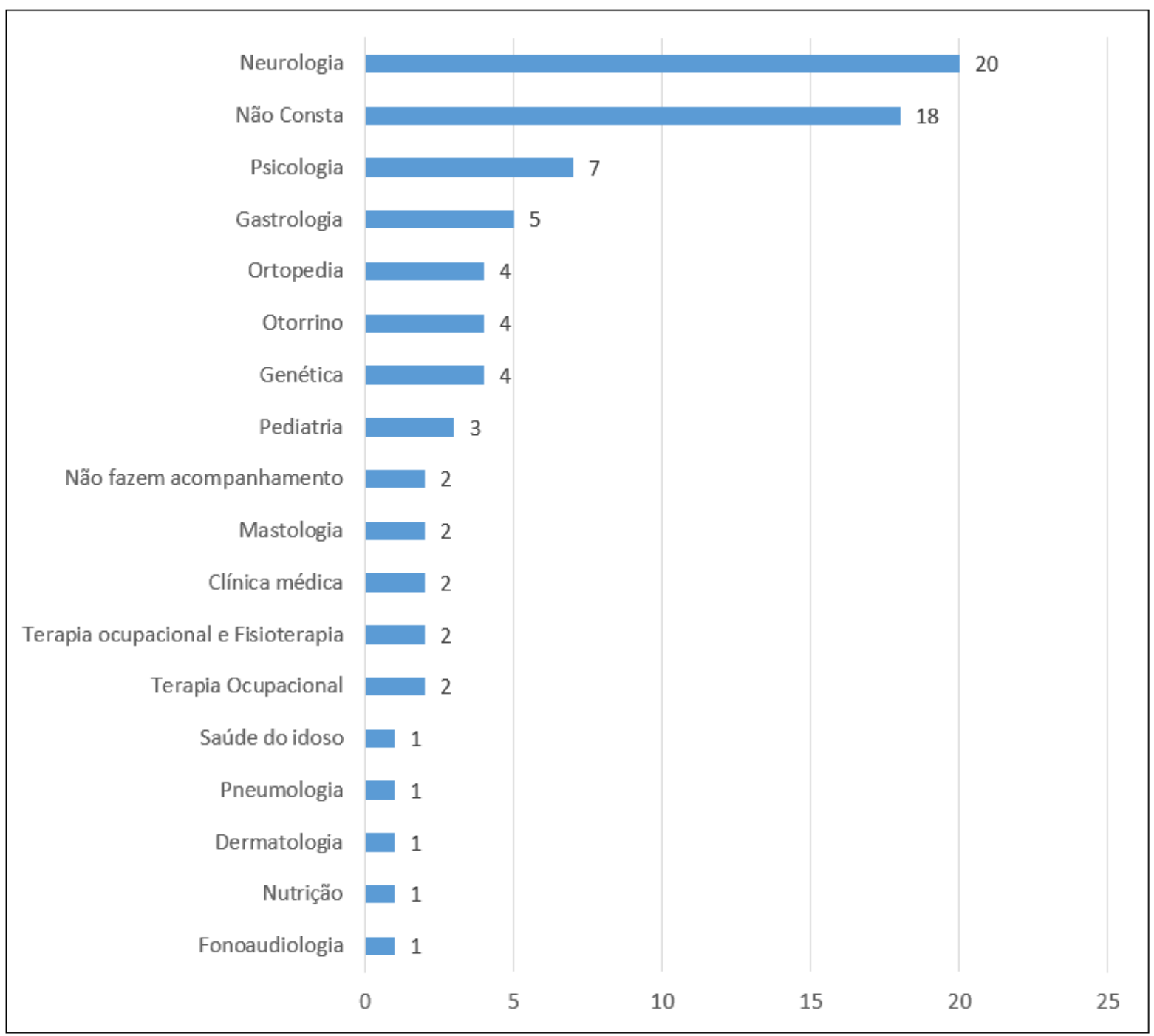

Fonte: Dati di ricerca.

RC: 79876

Disponível em: https://www.nucleodoconhecimento.com.br/salute/ambulatorio-di- 
Le diagnosi erano diversificate. Raramente erano visti in isolamento e erano spesso associati ad un'altra condizione. Nella clinica ambulatoriale GM, c'era una leggera prevalenza di ritardo dello sviluppo neuropsicomotorio (ADNPM), con 8 record e, in soli 2 casi, I'ADNPM era l'unica malattia diagnosticata nel paziente. Negli altri 6, era associato a breve statura e dismorfia (3), ipotiroidismo congenito (1) e pubertà precoce (2). Sono state trovate alcune sindromi, sindrome dismorfica (3), sindrome di Noonan (3), sindrome di Cornelia Lange (2), sindrome di Sotos (2), sindrome di Charge (1), sindrome di Marfan (1), sindrome di Aarskog (1), Down (1), sindrome di Beckwith-Wiedermann (1), sindrome di Turner (1), sindrome di Prader-Willi (1), sindrome di Williams (1) e sindrome ipercinetica associata al disturbo da deficit di attenzione e iperattività (1). Sono stati trovati cinque casi di cromosoma s46, XY e altri 5 casi di disabilità intellettiva sindromica. Oltre a 4 registrazioni di indagini sull'embriopatia da virus zyka, 3 indagini su malattie familiari, di cui 2 tumori al seno nelle sorelle. Gli errori inborni del metabolismo rappresentavano 4 casi, così come la breve statura dell'ereditarietà autosomica dominante. Acondroplasia, X-fragile ed epilessia associata a deficit cognitivo sono stati 3 pazienti ciascuno. Inoltre, neoplasia endocrina multipla di tipo 1 e neoplasia endocrina multipla di tipo $2 \mathrm{~A}$, microtia unilaterale destra, megacolon congenito con alterazioni scheletriche, lipodistrofia parziale di tipo dunning con oligomenorrea, neuropatia ereditaria distale e neurofibromatosi presentata 1 caso ciascuno (Figura 5).

Altre condizioni riscontrate sono state: esostasi multipla (2), iperplasia surrenale congenita (2), acidemia metilmalonica (1), adrenoleucodistrofia legata all'X (1), alterazione del comportamento multifattoriale (1), artrogriposi da predominanza distale associato a segni brida (1), ritardo nello sviluppo sessuale a causa dell'ipogonadismo (1), bronchiectasi non fibrotica (1), malattia di Dohi (1), carenza di ormone della crescita con bassa densità minerale ossea con sindrome miastenica associata alla sindrome di Kearnes-Sayre (1), ipotiroidismo centrale con mielosio funicolare e microadenoma efesicolare (1), carcinoma tiroideo midollare (1), encefalopatia cronica non progressiva (1), fibrosi cistica (1), genodermatosi

RC: 79876

Disponível em: https://www.nucleodoconhecimento.com.br/salute/ambulatorio-di- 
associata a linfedema (1) e ipodistrofia (1) (Figura 5). Entro il periodo di raccolta dei dati, 10 pazienti erano ancora in fase di studio o senza diagnosi completata.

Figura 5: Distribuzione dei pazienti osservati presso la clinica ambulatoriale di genetica medica, mediante diagnosi.

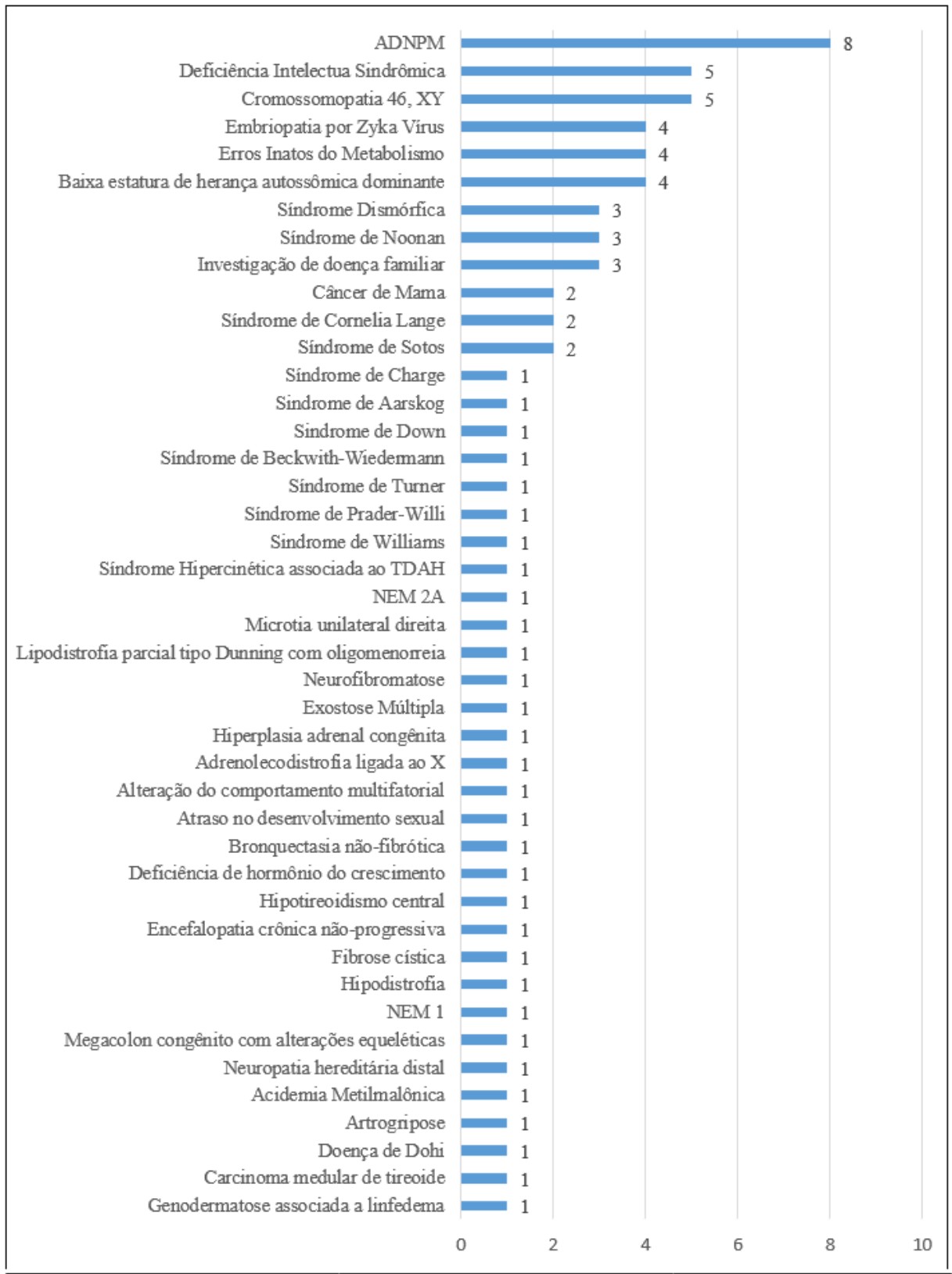

Fonte: Dati di ricerca.

RC: 79876

Disponível em: https://www.nucleodoconhecimento.com.br/salute/ambulatorio-di- 
In 42 cartelle cliniche, l'età alla diagnosi non era presente, 2 pazienti sono stati diagnosticati ancora nell'utero e altri 4 alla nascita. Ad altri 11 pazienti è stato diagnosticato un'età inferiore ai 2 anni; 13 pazienti, tra i 2 e i 9 anni; 18 durante l'adolescenza, con un numero più alto a 14 anni, con 6 diagnosi. Nella fascia di età adulta sono state diagnosticate 11 diagnosi e solo 1 in un paziente anziano a 63 anni (figura 6).

Figura 6: Distribuzione dei pazienti osservati presso la clinica ambulatoriale di genetica medica, per età alla diagnosi.

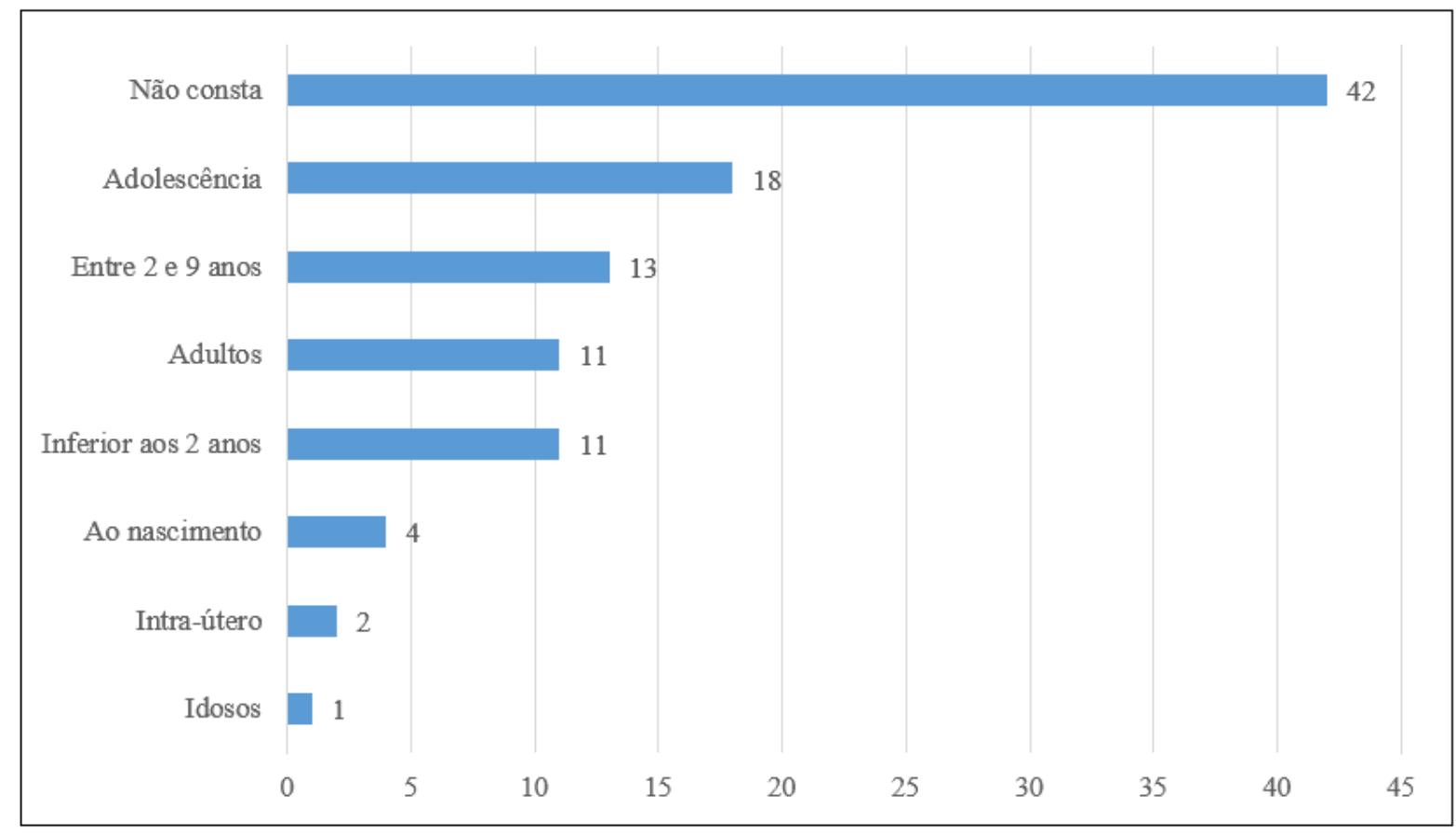

Fonte: Dati di ricerca.

Dei 101 pazienti, solo 16 avevano consulenza genetica e nei restanti 85 non c'era traccia di queste informazioni.

I pazienti sono stati seguiti più frequentemente da Endocrinologia, 21 record, seguiti da Neurologia con 20 pazienti e Psicologia seguita da 7 pazienti. Inoltre, sono stati monitorati 5 pazienti con Gastronomia, 4 con Genetica, Otorrino e Ortopedia, altri 3

RC: 79876

Disponível em: https://www.nucleodoconhecimento.com.br/salute/ambulatorio-di- 
con Pediatria, 2 con Terapia Occupazionale, 2 per Fisioterapia e Terapia Occupazionale contemporaneamente, 2 con la Clinica Medica, 2 con Mastologia, 1 con Logopedia, 1 con Nutrizione, 1 con Dermatologia, 1 con Pulmonologia e 1 con Salute Degli Anziani. Inoltre, 18 cartelle cliniche non hanno compilato la variabile e 2 non hanno dato seguito (figura 7).

Grafico 7: Distribuzione dei pazienti osservati presso la clinica ambulatoriale di genetica medica, per follow-up.

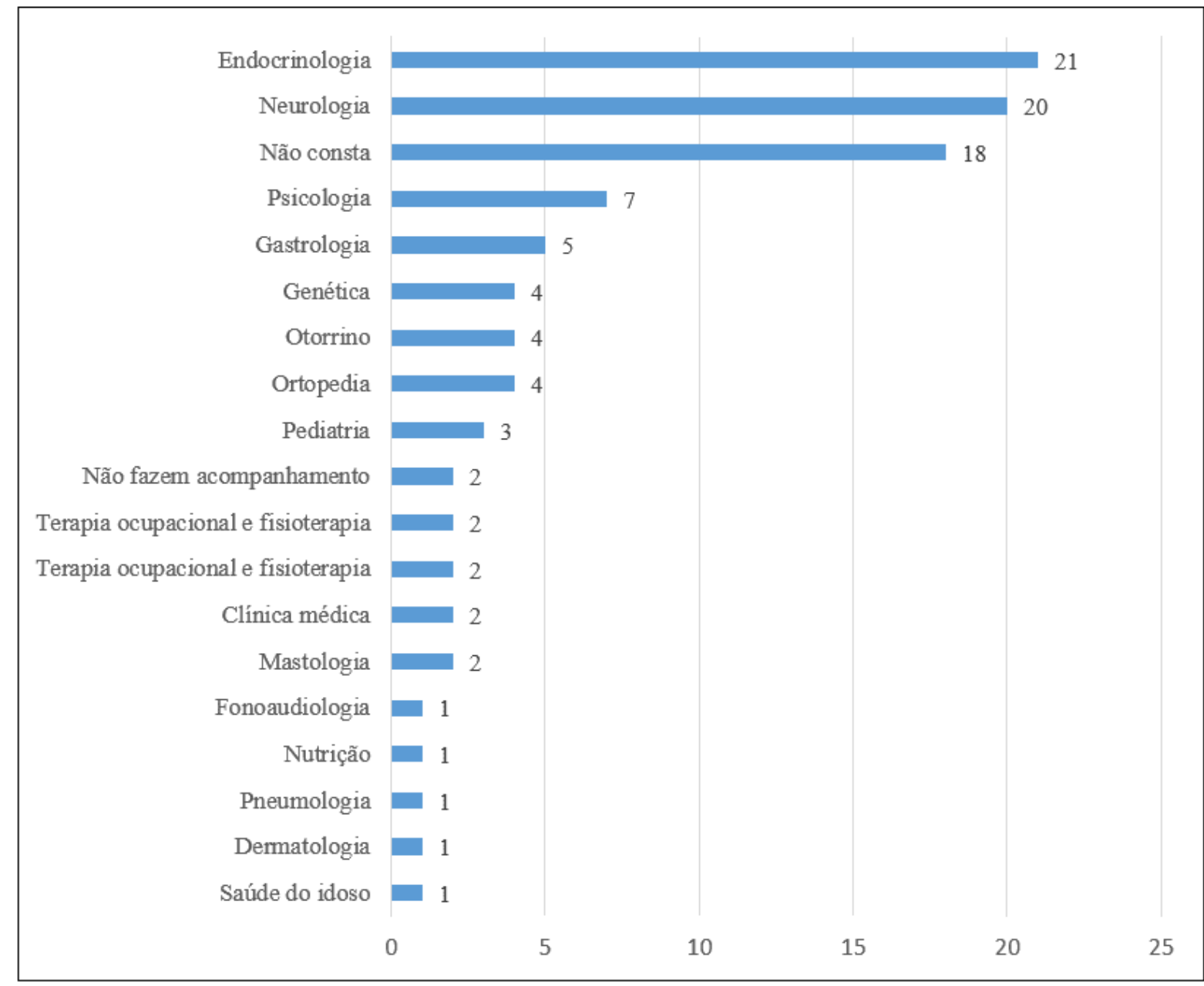

Fonte: Dati di ricerca.

RC: 79876

Disponível em: https://www.nucleodoconhecimento.com.br/salute/ambulatorio-digenetica 
Le informazioni sull'età della madre erano assenti nella maggior parte delle cartelle cliniche, non nel $61,38 \%$ del totale. Nelle cartelle cliniche in cui erano presenti le informazioni (38,62\%), le età materne tra i 16 e i 19 anni sono state del $7,92 \%$, mentre le età tra i 20 e i 59 anni erano nel $30,7 \%$ delle cartelle cliniche.

In 36 cartelle cliniche non c'erano informazioni sulle complicanze durante la gravidanza e in 25 casi non ci sono state complicazioni. Coloro che avevano (40) erano vari: infezione del tratto urinario (8), sanguinamento uterino (6), nascita pretermine (5), preeclampsia (4), ritardo nelle contrazioni di espulsione del feto (2), oligoidramnio (2), perina citossiatale (1), ciosi centrale (1), coliche nefrotiche (1), bambino intrappolato nel canale di parto (1), distacco placentare (1), dolore durante la gravidanza (1), consumo di alcol e fumo (1), ricovero in ospedale per la crisi dell'asma materna (1), morso canino (1), fumo, metrorragia e bambino con onfocele in USG - nello stesso paziente - (1), uso di colorante capillare e scolorimento dei peli del corpo (1), varicella (1) e virus materno Zyka (1).

\section{DISCUSSIONE}

Belém è un comune brasiliano dello stato del Pará, nella regione settentrionale del paese. È il comune più popoloso di Pará e il secondo nella regione settentrionale, con una popolazione di 1 abitanti.492.745 abitanti (INSTITUTO BRASILEIRO DE GEOGRAFIA E ESTATÍSTICA, 2020). La città ha una rete economica e di servizi compresa l'assistenza sanitaria - che serve le città della sua regione metropolitana.

Si ritiene che un medico genetista sia necessario ogni 100.000 abitanti, compresa la consulenza ambulatoriale, ospedaliera e genetica (CARVALHO et al., 2016; CONSELHO FEDERAL DE MEDICINA, 2020). Nello Stato di Pará, attualmente, solo 3 medici praticano la specialità del GM, secondo i dati del Consiglio Federale di Medicina (CONSELHO FEDERAL DE MEDICINA, 2020). Inoltre, tutti lavorano nella capitale Pará. Pertanto, si nota la scarsità del servizio, poiché la specialità avvantaggia l'intera collettività e accelera il sistema sanitario. Questo perché la

RC: 79876

Disponível em: https://www.nucleodoconhecimento.com.br/salute/ambulatorio-di- 
diagnosi precoce di malattie di origine genetica consente una migliore gestione dei pazienti e previene o riduce al minimo le possibili sequele e riduce anche i costi con possibili trattamenti più complessi.

Un altro dato rilevante evidenziato dallo studio è la mancanza di consulenza genetica da parte del team multidisciplinare che assiste il paziente, poiché solo circa il $16 \%$ dei pazienti aveva accesso a questo servizio, per gli altri, le informazioni erano assenti. Questo fatto può essere collegato alla scarsità del servizio, poiché non tutti i pazienti con malattia genetica hanno il servizio a loro disposizione, ed è necessario espandere la rete di assistenza a questo pubblico, data l'importanza della consulenza multiprofessionale, in particolare per quanto riguarda la prevenzione dei genotipi responsabili di malattie o difetti alla nascita (JORDE; CAREY; BAMSHAD, 2016).

Lo studio ha anche rivelato l'alto tasso di rinvii tra GM e altre specialità del Centro Medico in cui è stata condotta la ricerca. Le specialità con il maggior numero di rinvii erano endocrinologia e neurologia, oltre ad essere osservata il rinvio di GM ad altri professionisti, come psicologi e terapisti occupazionali. Essendo, essendo noto l'importanza dell'approccio multiprofessionale dei pazienti, poiché alcune malattie genetiche sono correlate a uno o più sistemi dell'organismo (BERTOLLO et al., 2013).

Per quanto riguarda l'età materna alla nascita del paziente, più della metà delle cartelle cliniche non conteneva le informazioni (62 cartelle cliniche), ma le cartelle cliniche che hanno presentato questi dati hanno rivelato che l'età della madre era superiore a 35 anni in 6 cartelle cliniche. Questi dati diventano rilevanti, poiché gli studi indicano un'associazione tra l'età materna avanzata e lo sviluppo di disturbi genetici. Gli studi rivelano che la prevalenza di complicanze genetiche nei feti delle madri con 20 anni è di 1 su 1.000 , mentre a 35 anni, questa proporzione raggiunge 1 donna incinta su 350. Confermare ancora una volta l'importanza della consulenza genetica (MENDES, 2017; BARBOZA et al., 2020).

RC: 79876

Disponível em: https://www.nucleodoconhecimento.com.br/salute/ambulatorio-di- 


\section{CONCLUSIONE}

L'elaborazione di un profilo per il paziente frequentato in una clinica ambulatoriale di Genetica Medica è estremamente importante, poiché, da queste conoscenze, diventa possibile identificare possibili guasti nel servizio fornito, oltre ad adattare il rapporto medico-paziente. Si sottolinea inoltre che la corretta accettazione ed esecuzione del trattamento e il follow-up di una malattia genetica dipendono da fattori personali e sociali, nonché dalla fase in cui la malattia sta contemplando la continuità del trattamento. Pertanto, la necessità di determinare il profilo del paziente in trattamento viene corroborata.

Così, i risultati del presente studio hanno dimostrato l'importanza di riempire in modo adeguato le cartelle cliniche del paziente frequentato presso la clinica ambulatoriale di Genetica Medica, al fine di evitare la perdita di informazioni cruciali, che possono aiutare a determinare la diagnosi o assistere nella conduzione del caso nel follow-up del paziente.

Un altro fattore importante evidenziato durante lo studio è la frequente sovrapposizione di diagnosi, poiché i pazienti di solito frequentano più di una patologia, è importante particolarizzare la cura, prestando attenzione a questo fatto, al fine di evitare possibili iatrogeniche e intercorrências, con interazioni farmacologiche durante il trattamento.

\section{RIFERIMENTI}

BARBOZA, B. P.; CALIL, C.; TRIGO, I. G. P. F.; ELLER, J. X.; SILVA, L. R.; VAZ, M. R.; ESTEVES, A. P. V. S. Idade materna avançada e seus desfechos. Cadernos da Medicina-UNIFESO, v. 2, n. 3, 2020.

RC: 79876

Disponível em: https://www.nucleodoconhecimento.com.br/salute/ambulatorio-di- 
Bertollo, E. M. G.; CASTRO, R; CINTRA, M. T. R.; PAVARINO, É. C. O processo de aconselhamento genético. Arq. Cienc. Saúde, v. 20, n. 1, p. 30-6, 2013.

BORGES-OSÓRIO, M. R; ROBINSON, W. M. Genética Humana. $3^{\text {a }}$ edição, Porto. Alegre, Editora Artmed, 2013.

CARVAlHo, A. C.; VIEIRA, E.; ALTENHOFEn, T. M. G.; JUNG, M. S. Perfil clínicoepidemiológico dos pacientes atendidos pelo Serviço de Genética Médica do Ambulatório Materno Infantil da Universidade do Sul de Santa Catarina. Arquivos Catarinenses de Medicina, v. 45, n. 2, p. 11-24, 2016.

CONSELHO FEDERAL DE MEDICINA. Disponível em: < https://sistemas.cfm.org.br/normas/arquivos/pareceres/BR/2018/17_2018.pdf > Acesso em 20 de março de 2020.

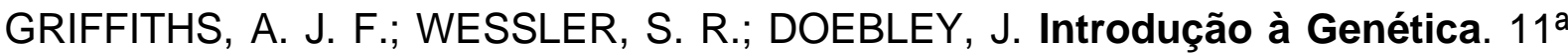
edição. Guanabara Saúde Didático, 2016.

INSTITUTO BRASILEIRO DE GEOGRAFIA E ESTATÍSTICA. Disponível em: $<$ https://www.ibge.gov.br/cidades-e-estados/pa/belem.html> Acesso em: 20 de março de 2020.

JORDE, L. B.; CAREY, J. C.; BAMSHAD, M. J.; Genética Médica. 5aㅗ edição, Rio de Janeiro, Editora Elsevier. 2016.

LOBO, I. Environmental influencies on gene expression. Nature Education 1(1):39, 2008

MENDES, C. C. Estudo genético e epigenético de fatores de risco materno para a síndrome de Down. 2017. 32 f. Tese (Programa de Pós-Graduação em Ciências

RC: 79876

Disponível em: https://www.nucleodoconhecimento.com.br/salute/ambulatorio-di- 
da Saúde) - Faculdade de Medicina de São José do Rio Preto, São José do Rio Preto.

NUSSBAUM, R. L.; MCINNES, R. R.; HUNTINGTON, F. W.; THOMPSON, M. W. Thompson \& Thompson. Genetics in Medicine. Philadelphia: Elsevier. 2016.

SOCIEDADE BRASILEIRA DE GENÉTICA MÉDICA. Disponível em: <http://www.sbgm.org.br>. Acesso em: 20 março, 2020.

ZANELLA, J.; TERENCIO, M. L. Genética clínica: investigação dos padrões de herdabilidade de doenças humanas-mutações genéticas e cromossômicas. Artigo completo publicado em: Anais do V Encontro de Iniciação Científica e I Encontro Anual de Iniciação ao Desenvolvimento Tecnológico e Inovação - EICTI. 2016.

Inviato: Marzo 2021.

Approvato: Marzo 2021.

RC: 79876

Disponível em: https://www.nucleodoconhecimento.com.br/salute/ambulatorio-di- 